\title{
STUDY THE EFFICACY OF CHRONIC INTERMITTENT INTRAVENOUS INSULIN INFUSION THERAPY OVER AND ABOVE CONVENTIONAL TREATMENT IN PATIENTS OF DIABETIC AUTONOMIC NEUROPATHY
}

\author{
MAHESHWARI PK ${ }^{1}$, PRABHAT AGRAWAL ${ }^{1}$, PRASHANT PRAKASH $^{2 *}$, SINGH AK ${ }^{1}$, SAURABH BANSAL $^{1}$, \\ DEEPAK PUROHIT ${ }^{1}$, SHWETA AGARWAL ${ }^{3}$
}

\begin{abstract}
${ }^{1}$ Department of Medicine, Sarojini Naidu Medical College, Agra, Uttar Pradesh, India. ${ }^{2}$ Department of Medicine, Unit Head Pulmonary Medicine, Sarojini Naidu Medical College, Agra, Uttar Pradesh, India. ${ }^{3}$ Department of Pathology, Sarojini Naidu Medical College, Agra, Uttar Pradesh, India. Email: dr.prashantprakash@gmail.com

Received: 03 March 2017, Revised and Accepted: 22 May 2018
\end{abstract}

ABSTRACT

Objective: This study aims to emphasize the importance of chronic intermittent intravenous insulin infusion therapy (CIIIT) over and above conventional treatment in patients with diabetic autonomic neuropathy.

Methods: In our study, we had 50 patients; most of the patients were in the age group of 30-50 years and sex ratio for male:female was 3:2. The patients were divided into two groups. One group which received monthly CIIIT for $8 \mathrm{~h}$ per month for 6 months (Group-1) whereas other group did not receive it (Group-2). However, both groups continued their old treatment (oral hypoglycemic or subcutaneous insulin).

Results: On 12-month follow-up, in Group-1, palpitation improved in 16 of 17 (94.1\%), giddiness improved in 12 of 20 (60.0\%), constipation improved in 7 of 12 (58.3\%), diarrhea improved in 7 of 8 (87.5\%), erectile dysfunction improved in 3 of 5 (60.0\%), abdominal fullness improved in 12 of $17(70.5 \%)$, and abnormal sweating improved in 6 of 20 (30\%). In comparison to this, in Group 2, palpitation improved in 6 of 19 (31.5\%), giddiness improved in 2 of 15 (13.3\%), constipation improved in 3 of 13 (23.0\%), diarrhea improved in 3 of 7 (42.8\%), erectile dysfunction improved in 3 of 5 (20.0\%), abdominal fullness improved in 4 of 13 (13.7\%), and abnormal sweating improved in 5 of 17 (29.4\%).

Conclusion: At the conclusion of our study, we found that patients, who were previously refractory to conventional pharmacotherapy, showed a considerable response to CIIIT with most patients giving a history of improvement in their symptoms after therapy and during follow-up. Therefore, it is recommended that patients presenting with complaints of diabetic autonomic neuropathy should be managed by CIIIT rather than the conventional pharmacological therapies.

Keywords: Chronic intermittent intravenous insulin therapy, Diabetes mellitus, Diabetic autonomic neuropathy.

(c) 2018 The Authors. Published by Innovare Academic Sciences Pvt Ltd. This is an open access article under the CC BY license (http://creativecommons. org/licenses/by/4. 0/) DOI: http://dx.doi.org/10.22159/ajpcr.2018.v11i9.25613

\section{INTRODUCTION}

Diabetes mellitus is the most common endocrine disease worldwide. It is a syndrome characterized by chronic hyperglycemia and disturbances of carbohydrate, fat and protein metabolism associated with absolute or relative deficiencies in insulin secretion and/or insulin action. Its long-term complications lead to significant morbidity and mortality. Autonomic neuropathy is one such neuropathy, it is estimated that $8 \%$ of diabetics have diabetic autonomic neuropathic complications at the time of diagnosis and $50 \%$ develop them within 25 years of diagnosis [1]. Oral supplementation of $\alpha$-lipoic acid was found to be effective in improving motor nerve conduction of the upper and lower extremities in patients with diabetic peripheral neuropathy [2].

Clinical Manifestations of autonomic neuropathies [3] include the following:

1. Cardiovascular: Alterations in skin blood flow, cardiac denervation, painless myocardial infarction, heat intolerance, orthostatic hypotension, tachycardia, and exercise intolerance

2. Gastrointestinal: Constipation, diarrhea, esophageal dysfunction, fecal incontinence, and gastroparesis diabeticorum

3. Genitourinary: Cystopathy, erectile dysfunction, neurogenic bladder, and retrograde ejaculation

4. Metabolic: Hypoglycemia unawareness and hypoglycemia unresponsiveness

5. Pupillary: Argyll Robertson type and decreased diameter of darkadapted pupil
6. Sweating disturbances: Areas of symmetrical anhidrosis and gustatory sweating.

CIIIT, also known as hepatic activation therapy or metabolic activation therapy or pulsatile intravenous insulin therapy, is an experimental and investigational tool for the management of diabetes mellitus and all other indications because its clinical value is not yet established. CIIIT is an insulin therapy that delivers insulin in a pulsatile fashion and supposedly achieves physiologic insulin concentration in the portal vein [4].

CIIIT is performed as a monthly procedure in conjunction with daily intensive subcutaneous insulin therapy or oral hypoglycemic agents. A CIIIT session entails a 6-8 h period in an inpatient or outpatient setting. The patient receives intermittent pulses of insulin through an intravenous catheter in a peripheral vein in hand or arm. The intermittent pulses are controlled by the frequent monitoring of blood glucose levels and the timing of glucose load ingested by the patient.

CIIIT has been reported to (1) improve significantly glycemic control while reducing the incidence of hypoglycemic events, (2) improve hypertension control, (3) slow the progression of overt diabetic nephropathy (DN), and (4) reverse some manifestations of diabetic autonomic neuropathy including abnormal circadian blood pressure (BP) pattern, severe postural hypotension, and hypoglycemia unawareness [4]. 


\section{METHOD}

This prospective study was carried out from june 1, 2016 to May 31, 2017 in S.N. Medical College, Agra, after institutional ethics committee approval. Fifty patients of diabetes mellitus with different duration of diabetes, attending the diabetic clinics, OPD, and indoors of the Post Graduate Department of Medicine, S.N. Medical College, Agra, constituted the material for the present study. Informed information cum consent form containing all information about name, age, sex, residence, etc., of the subject and information of the study and commitment for monthly requirements associated with one group of patients will be obtained from every patient in advance. Cases were having signs or symptoms or both or investigations suggestive of autonomic neuropathy.

CIIIT was given in a pulsatile manner (once in every 30 days) by adding $8 \mathrm{U}$ of regular insulin to $500 \mathrm{ml}$ of dextrose and infusing slowly with regular blood sugar charting. The insulin dose was the same for all patients as all of them were euglycemic. The patient receives intermittent pulses of insulin through an intravenous catheter in a peripheral vein in the hand or arm. The intermittent pulses are controlled by the frequent monitoring of blood glucose levels and the timing of glucose load ingested by the patient.

Exclusion criteria for the study include the following:

- Patients with diabetes mellitus in whom blood sugar is uncontrolled (glycosylated hemoglobin [HbA1c] > 7.5\%).

- Other conditions causing autonomic neuropathy excluded such as hypertension, ischemic heart disease, valvular heart disease, hepatic cirrhosis, prostatic enlargement, heart failure, urinary tract infection, fever, secondary hyperglycemic conditions such as Cushing syndrome, pancreatitis, acromegaly, pheochromocytoma, and hyperthyroidism

- The patients who had participated in the past 30 days or currently participating in another trial.

- Any patient who was found to have another alternative explanation for his symptoms (e.g., mechanical obstruction of the gastrointestinal tract) was excluded from the study.

\section{Statistical analysis}

Descriptive statistical analysis of data such as frequency and percentages were used for categorical variables. The mean and standard deviation were used for describing continuous variables. Inferential statistical tools such as Chi-square and Student's t-tests were used. $\mathrm{P}<0.05$ were considered to be statistically significant.

The symptoms were compared at the commencement of the study, after six sessions of CIIIT (i.e., after 6 months) and finally after 12 months. This was done by asking the patient to grade their symptoms on the

Table 1: Age and sex distribution of cases

\begin{tabular}{lccc}
\hline Age group & Male (\%) & Female (\%) & Total (\%) \\
\hline $31-30$ & $5(38.4)$ & $8(61.5)$ & $13(52)$ \\
$41-50$ & $2(16.6)$ & $10(83.3)$ & $12(48)$ \\
$51-60$ & $0(0)$ & $0(0)$ & $0(0)$ \\
\hline
\end{tabular}

visual analog scale (VAS) on each visit. The patients were subjected to detailed history, and clinical examination and the following symptoms were noted: Palpitation, giddiness, constipation, diarrhea, erectile dysfunction, abdominal fullness, and abnormal sweating were graded on the VAS in the range of $0-10$ on the basis of severity. Routine investigations including blood sugar (fasting and postprandial), ultrasonography abdomen, urine ketodiastix (to rule out diabetic ketoacidosis), serum creatinine, serum glutamic pyruvic transaminase, and $\mathrm{HbA1c}$ at the beginning and end of the study were also done for all the patients.

\section{RESULTS}

The findings of this work are being discussed under the headings of demographic profile, clinical features, and discussion.

\section{Demographic profile}

The majority of our cases were of Type 2 diabetes mellitus (90\%). The mean age of our study was 45 years with the minimum age of 31 years and the maximum age of 60 years. The majority of our patients (53\%) were in the age group of 31-50 years. The male-to-female ratio in our study was 3:2 (males-30; and females-20) (Table 1).

\section{Clinical features}

Among presenting symptoms, palpitation was the most common (72\%) followed by giddiness (70\%), abnormal sweating (64\%), abdominal fullness (60\%) and constipation (50\%), erectile dysfunction (40\%), and diarrhea (30\%). Most of the patients presented with four or five symptoms $(40 \%$ each), followed by three symptoms $(24 \%)$. About $8 \%$ of patients had five of the seven symptoms. No patient had either one or all the seven symptoms. Giddiness was the severest symptom of the seven with a mean VAS score of 8 , followed by palpitation which had a mean VAS score of 7 . These were followed by diarrhea, constipation, abdominal fullness, and abnormal sweating which had mean VAS scores of 6.75, 6.37, 5.20 , and 5.10, respectively. Erectile dysfunction was the least severe symptom with a VAS score of 5.00 (Table 2).

\section{DISCUSSION}

All the symptoms, which were previously refractory to the patient conventional pharmacotherapy, showed a considerable response to our therapy with CIIIT with most patients giving a history of improvement in their symptoms after therapy and during follow-up. The VAS scores showed a significant decline in all symptoms. However, no patient gave a history of complete recovery, with nearly all of them complaining of at least some residual symptoms. The mean $\mathrm{HbA1c}$ at starting of the study was 7.25 and 7.15 in control and CIIIT group. After 6 months of followup, the mean HbA1c was 7.15 and 6.95, respectively. After 12 months of follow-up, the mean HbA1c was 7.16 and 6.81, respectively, in control and CIIIT group, which was statistically significant $(\mathrm{p}<0.0001)$.

In the literature available to us, several studies on various diabetic complications have supported its use for the same. A study conducted by Maheshwari et al., [5] studied the effect of CIIIT on diabetic gastroparesis found the clear-cut benefit of CIIIT over conventional pharmacological therapy such as metoclopramide, domperidone, levosulpiride, and

Table 2: Symptomatic improvement (mean values of VAS)

\begin{tabular}{lccc}
\hline Symptoms & At presentation & After 6 months & After 12 months \\
\hline Giddiness & 8.00 & 4.48 & 4.01 \\
Palpitation & 7.00 & 3.11 & 2.98 \\
Diarrhea & 6.75 & 4.51 & 3.58 \\
Constipation & 6.37 & 3.58 & 3.10 \\
Abdominal fullness & 5.20 & 2.11 & 2.00 \\
Abnormal sweating & 5.10 & 3.63 & 3.58 \\
Erectile dysfunction & 5.00 & 2.25 & 2.01 \\
\hline
\end{tabular}


erythromycin. A study by Aoki et al., (1995) [6] studied the effect of long-term intermittent intravenous insulin therapy on Type 1 diabetes mellitus and found that it not only decreased the mean $\mathrm{HbA} 1 \mathrm{c}$ values from 8.5 to 7.0 but also decreased the occurrence of both major and minor hypoglycemic episodes. A decrease in the antihypertensive dosage was also observed in the same study, and it indicated that CIIIT markedly improves BP control in patients with IDDM and hypertension, probably by improving the vascular smooth muscle tone. Aoki et al., (2001) [6], Aoki et al. [7], the diabetes control and complications trial research group (1993) [8] also concluded through a retrospective, longitudinal study that CIIIT could successfully stabilize renal function in patients with DN. Aoki et al., [9] have demonstrated a blunted diurnal variation in BP in patients with diabetic autonomic neuropathy which leads to decreased target organ damage. Daily et al., [10] through a multi-institutional prospective, randomized, controlled study evaluated the effect of CIIIT in patients with DN and found that the treatment group (CIIIT) patients had a statistically significant improvement in renal function as compared to the control group. CIIIT, when given to patients with diabetic autonomic neuropathy not only improves their glycemic control but also bring about symptomatic relief and also prolong the development of other micro and macrovascular complications of diabetes mellitus, also symptomatic improvement by other poorly understood mechanisms. This finding suggests that treatment with coenzyme Q10 or rosuvastatin showed a significant neuroprotective effect against STZ-nicotinamide-induced diabetic neuropathy. However, concomitant administration of both showed a better neuroprotective effect than coenzyme Q10 or rosuvastatin alone treatment [11].

\section{CONCLUSION}

All our patients treated with CIIIT showed improvement in their symptomatology. They did not develop hypoglycemia and also showed sustained benefit. The sustainability of the benefit over the next 2 years is under the study. Therefore, it is recommended that patients presenting with complaints of diabetic autonomic neuropathy should be managed by CIIIT rather than the conventional pharmacological therapies. Further work with a longer study with many more patients is required to properly quantify these changes and understand the exact mechanism how CIIIT.

\section{ACKNOWLEDGMENT}

The authors are grateful to all faculty members of P.G. Department of medicine, S.N. Medical Agra, Uttar Pradesh, India, for their contribution.

\section{CONFLICTS OF INTEREST}

There are no conflicts of interest.

\section{AUTHORS CONTRIBUTION}

PK.M. implemented the model and performed all the simulations in this manuscript. PK.M., P.A., P.P., AK.S., S.B., D.P., S.A. contributed to the interpretation of simulations. P.K.M., P.A., P.P., AK.S., S.B., D.P., S.A. contributed the conception and design of the work and in developing the manuscript.

\section{REFERENCES}

1. Lakhiar MA, Shahbaz NN, Bughio AH, Prakash J. Frequency of peripheral neuropathy in newly diagnosed patients of diabetes mellitus iion clinical and electrophysiological basis. Pak J Neurol Sci (PJNS) 2014;9:31-5

2. Anandvijayakumar PR, Kalshetti SM, Bhatt JK. Supplementation of $\alpha$-lipoic acid in diabetic peripheral neuropathy: A prospective open label randomized controlled trial. Int J Pharm Pharm Sci 2014;6:90-3. Available from: https://www.innovareacademics.in/journals/index.php/ ijpps/article/view/1247/862. [Last Accessed on 2018 Apr12].

3. Kronenberg HM, Melmed S, Polonsky KS, Larsen PR. Complication of Diabetes Mellitus Autonomic Neuropathy in Williams Textbook of Endocrinology, 11 $1^{\text {th }}$ d. Philadelphia, PA: Saunders Elsevier; 2008. p. 1464-70.

4. Aoki TT, Grecu EO, Arcangeli MA, Benbarka MM, Prescott P, Ahn JH. Chronic intermittent intravenous insulin therapy: A new frontier in diabetes therapy. Diabetes Technol Ther 2001;3:102-17.

5. Maheshwari PK, Bansal M, Agrawal P, Agarwal A. Int J Basic Appl Med Sci 2016;6:36-40: An Open Access, International Journal. Available from: http://www.cibtech.org/jms.htm.

6. Aoki TT, Grecu EO, Gollapudi GM, Barber RA, Arcangeli MA, Benbarka MM, et al. Effect of intensive insulin therapy on progression of overt nephropathy in patients with Type I diabetes mellitus. Endocr Pract 1999;5:174-8.

7. Aoki TT, Benbarka MM, Okimura MC, Arcangeli MA, Walter RM, Wilson LD, et al. Long-term intermittent intravenous insulin therapy and Type I diabetes mellitus. Lancet 1993;342:515-8.

8. The Diabetes Control and Complications Trial Research Group. The effect of intensive treatment of diabetes on the development and progression of long-term complications in insulin dependent diabetes mellitus. N Engl J Med 1993;329:977-86.

9. Aoki, TT, Grecu EO, Arcangeli MA, Meisenheimer R. Effect of intensive insulin therapy on abnormal circadian blood pressure pattern in patients with Type 1 diabetes mellitus. Online J Curr Clin Trials 1995; 199:515-8.

10. Daily GE, Boden GH, Creech RH, Johnson DG, Gleason RE, Kennedy FP, et al. Effects of pulsatile intravenous insulin therapy on the progression of diabetic nephropathy. Metabolism 2003;49:1491-5.

11. Maheshwari RA, Balaraman R, Sen AK, Seth AK. Effect of coenzyme Q10 alone and its combination with rosuvastatin on streptozotocinnicotinamide induced diabetic neuropathy in rats. Int J Pharm Pharm Sci 2014;46:296-9. Available from: https://www.innovareacademics. in/journals/index.php/ijpps/article/view/3802/1721. [Last Accessed on 2018 Apr12]. 